\title{
MARCINKIEWICZ INTERPOLATION THEOREMS FOR ORLICZ AND LORENTZ GAMMA SPACES
}

\author{
Ron Kerman, Colin Phipps, And Luboš Pick
}

\begin{abstract}
Fix the indices $\alpha$ and $\beta, 1<\alpha<\beta<\infty$, and suppose $\varrho$ is an Orlicz gauge or Lorentz gamma norm on the real-valued functions on a set $X$ which are measurable with respect to a $\sigma$-finite measure $\mu$ on it. Set

$$
M(\gamma, X):=\left\{f: X \rightarrow \mathbb{R} \text { with } \sup _{\lambda>0} \lambda \mu(\{x \in X:|f(x)|>\lambda\})^{\frac{1}{\gamma}}<\infty\right\},
$$

$\gamma=\alpha, \beta$. In this paper we obtain, as a special case, simple criteria to guarantee that a linear operator $T$ satisfies $T: L_{\varrho}(X) \rightarrow L_{\varrho}(X)$, whenever $T: M(\alpha, X) \rightarrow M(\alpha, X)$ and $T: M(\beta, X) \rightarrow M(\beta, X)$.
\end{abstract}

2010 Mathematics Subject Classification: Primary: 46E35; Secondary: 46E30.

Key words: Supremum operators, Sobolev imbeddings, optimality, rearrangementinvariant norms.

\section{Introduction}

A generalization of the Marcinkiewicz interpolation theorem to Orlicz spaces contains the conditions

$$
\begin{aligned}
& \int_{b^{-1}}^{t} \frac{A(s)}{s^{\alpha+1}} d s \leq \frac{A(K t)}{t^{\alpha}}, \\
& \int_{t}^{\infty} \frac{A(s)}{s^{\beta+1}} d s \leq \frac{A(K t)}{t^{\beta}},
\end{aligned}
$$

where $1<\alpha<\beta<\infty, 0<b \leq \infty, A$ is a Young function and $K>0$ is a constant independent of $t \in\left(b^{-1}, \infty\right)$; see [20, Vol. II, Chapter XII, Theorem 4.22]. One of the consequences of a principal result of this paper is that if $L_{A}=L_{A}(X)$ is an Orlicz space defined with respect

This research was supported in part by NSERC grant A4021, an USRA grant from NSERC, grant MSM 0021620839 of the Czech Ministry of Education, grants 201/07/0388 and 201/08/0383 of the Grant Agency of the Czech Republic, NATO grant PST.CLG.978798, Leverhulme Trust Grant n.F/00407/E and by the Nečas Center for Mathematical Modelling project no. LC06052 financed by the Czech Ministry of Education. 
to a $\sigma$-finite measure $\mu$ on $X, \mu(X)=b$, then the conditions (1.1) are necessary and sufficient for $L_{A}$ to be an interpolation space between the Marcinkiewicz spaces $M(\alpha)=M(\alpha, X)$ and $M(\beta)=M(\beta, X)$. Recall that $f \in M(\alpha)$, say, is equivalent to

$$
\varrho_{M(\alpha, X)}(f):=\sup _{\lambda>0} \lambda \mu_{f}(\lambda)^{\frac{1}{\alpha}}<\infty,
$$

in which

$$
\mu_{f}(\lambda):=\mu(\{x \in X:|f(x)|>\lambda\}) .
$$

We will work in the general setting of rearrangement-invariant (r.i.) norms, $\varrho$, on the class $\mathfrak{M}(X)$ of $\mu$-measurable functions on $X$. Such a norm determines an r.i. space

$$
L_{\varrho}=L_{\varrho}(X):=\{f \in \mathfrak{M}(X): \varrho(|f|)<\infty\} .
$$

See Section 2 below for details. We only mention here that the key property of an r.i. norm is

$$
\varrho(f)=\varrho(g)
$$

whenever $f$ and $g$ are equimeasurable, in the sense that $\mu_{f}=\mu_{g}$.

Two families of r.i. norms will be of special interest to us, namely, the Orlicz gauge norms and the Lorentz gamma norms. The former norms are defined in terms of a Young function, $A$, by

$$
\varrho_{A}(f):=\inf \left\{\lambda>0: \int_{X} A\left(\frac{|f(x)|}{\lambda}\right) d x \leq 1\right\} .
$$

The latter norms are given in terms of an index $p, 1<p<\infty$, and a positive, locally integrable (weight) function, $\phi$, on $I_{b}=(0, b), b=\mu(X)$, by

$$
\varrho_{p, \phi}(f):=\left[\int_{I_{b}} f^{* *}(t)^{p} \phi(t) d t\right]^{\frac{1}{p}}, \quad f \in \mathfrak{M}(X)
$$

here,

$$
f^{* *}(t):=t^{-1} \int_{0}^{t} f^{*}(s) d s
$$

with

$$
f^{*}=\mu_{f}^{-1},
$$

the inverse being in a generalized sense; again, see Section 2 below. We require

$$
\int_{1}^{\infty} \phi(t) t^{-p} d t<\infty, \text { when } b=\infty \text {, and } \int_{I_{b}} \phi(t) t^{-p} d t=\infty \text {, when } b<\infty
$$


otherwise, $\Gamma_{p, \phi}:=L_{\varrho_{p, \phi}}$ would consist only of the zero function in the first case and would be identical to the space $L_{1}=L_{1}\left(I_{b}\right)$ of Lebesgue-integrable functions on $I_{b}$ in the second case. Such weights $\phi$ will be called nontrivial.

We first state a result in which the boundedness of certain operators $T$ is asserted to follow from that of the supremum operators, $S_{\alpha}$ and $T_{\beta}$, $\alpha, \beta>1$, defined at Lebesgue-measurable $f$ on $I_{b}$ and $t \in I_{b}$ by

$$
\begin{aligned}
& \left(S_{\alpha} f\right)(t):=t^{-\frac{1}{\alpha}} \sup _{0<s \leq t} s^{\frac{1}{\alpha}} f^{*}(s), \\
& \left(T_{\beta} f\right)(t):=t^{-\frac{1}{\beta}} \sup _{t \leq s<b} s^{\frac{1}{\beta}} f^{*}(s),
\end{aligned}
$$

respectively. This result is proved in a more general setting by Dmitriev and Kreı̆n [7], though the authors state that an earlier version in our context is due to Peetre. We give here a new proof (see Section 3) that emphasizes the role of the operators $S_{\alpha}$ and $T_{\beta}$, which role is only implicit in the work of the previous authors.

Theorem 1.1 (Dmitriev-Krĕn-Peetre). Let $\left(X_{1}, \mu_{1}\right)$ and $\left(X_{2}, \mu_{2}\right)$ be $\sigma$-finite measure spaces for which $\mu_{1}\left(X_{1}\right)=\mu_{2}\left(X_{2}\right)=b$. Suppose the quasilinear operator $T$ satisfies

$$
T: M\left(\alpha, X_{1}\right) \rightarrow M\left(\alpha, X_{2}\right) \text { and } T: M\left(\beta, X_{1}\right) \rightarrow M\left(\beta, X_{2}\right)
$$

for indices $\alpha$ and $\beta$, with $1<\alpha<\beta<\infty$. Define the r.i. norms, $\varrho_{i}$, on $\mathfrak{M}\left(X_{i}\right)$ in terms of given r.i. norms, $\bar{\varrho}_{i}$, on $\mathfrak{M}\left(I_{b}\right)$ by

$$
\varrho_{i}(f)=\bar{\varrho}_{i}\left(f^{*}\right)
$$

and suppose

$$
M\left(\alpha, X_{i}\right) \cap M\left(\beta, X_{i}\right) \subset L_{\varrho_{i}}\left(X_{i}\right) \subset M\left(\alpha, X_{i}\right)+M\left(\beta, X_{i}\right), \quad i=1,2 .
$$

Then,

$$
T: L_{\varrho_{1}}\left(X_{1}\right) \rightarrow L_{\varrho_{2}}\left(X_{2}\right)
$$

whenever

$$
S_{\alpha}: L_{\bar{\varrho}_{1}}\left(I_{b}\right) \rightarrow L_{\bar{\varrho}_{2}}\left(I_{b}\right) \text { and } T_{\beta}: L_{\bar{\varrho}_{1}}\left(I_{b}\right) \rightarrow L_{\bar{\varrho}_{2}}\left(I_{b}\right) .
$$

Our paper is devoted to obtaining simple criteria to guarantee (1.2) when $\varrho_{1}$ and $\varrho_{2}$ are both Orlicz gauge norms or both Lorentz gamma norms. These criteria, asserting that it suffices to test the boundedness of $S_{\alpha}$ and $T_{\beta}$ on characteristic functions of sets, are given in Theorems A and $B$, which we now state. 
Theorem A. Let $\left(X_{1}, \mu_{1}\right)$ and $\left(X_{2}, \mu_{2}\right)$ be $\sigma$-finite measure spaces with $\mu_{1}\left(X_{1}\right)=\mu_{2}\left(X_{2}\right)=b$. Fix the indices $\alpha$ and $\beta, 1<\alpha<\beta<\infty$. Suppose $A_{1}$ and $A_{2}$ are Young functions satisfying

$$
M\left(\alpha, X_{i}\right) \cap M\left(\beta, X_{i}\right) \subset L_{A_{i}}\left(X_{i}\right) \subset M\left(\alpha, X_{i}\right)+M\left(\beta, X_{i}\right), \quad i=1,2 .
$$

Assume, in addition, that $t^{-\frac{1}{\alpha}} \notin L_{A_{2}}\left(I_{b}\right)$,

$$
A_{2}(t)=0, \quad t \in I_{b^{-1}},
$$

when $b<\infty$,

when $b=\infty$ and

$$
\int_{0}^{1} A_{2}(t) t^{-1-\alpha} d t<\infty
$$

$$
\int_{1}^{\infty} A_{2}(t) t^{-1-\beta} d t<\infty
$$

for all $b$.

Then, given any quasilinear operator $T$ such that

$$
T: M\left(\alpha, X_{1}\right) \rightarrow M\left(\alpha, X_{2}\right) \text { and } T: M\left(\beta, X_{1}\right) \rightarrow M\left(\beta, X_{2}\right),
$$

one has

$$
T: L_{A_{1}}\left(X_{1}\right) \rightarrow L_{A_{2}}\left(X_{2}\right),
$$

whenever

$$
\begin{gathered}
\int_{b^{-1}}^{t} \frac{A_{2}(s)}{s^{\alpha+1}} d s \leq \frac{A_{1}(K t)}{t^{\alpha}}, \\
\int_{t}^{\infty} \frac{A_{2}(s)}{s^{\beta+1}} d s \leq \frac{A_{1}(K t)}{t^{\beta}},
\end{gathered}
$$

the constant $K>0$ being independent of $t \in\left(b^{-1}, \infty\right)$.

In particular, the first condition in (1.3) is necessary and sufficient in order that

$$
S_{\alpha}: L_{A_{1}}\left(I_{b}\right) \rightarrow L_{A_{2}}\left(I_{b}\right),
$$

while the second condition is necessary and sufficient for

$$
T_{\beta}: L_{A_{1}}\left(I_{b}\right) \rightarrow L_{A_{2}}\left(I_{b}\right) .
$$

Theorem B. Let $\left(X_{1}, \mu_{1}\right)$ and $\left(X_{2}, \mu_{2}\right)$ be $\sigma$-finite measure spaces for which $\mu_{1}\left(X_{1}\right)=\mu_{2}\left(X_{2}\right)=b$. Fix the indices $\alpha$ and $\beta$, with $1<\alpha<$ $\beta<\infty$. Suppose the index $p, 1<p<\infty$, and the nontrivial weight functions, $\phi_{1}$ and $\phi_{2}$, are such that

$$
M\left(\alpha, X_{i}\right) \cap M\left(\beta, X_{i}\right) \subset \Gamma_{p, \phi_{i}}\left(X_{i}\right) \subset M\left(\alpha, X_{i}\right)+M\left(\beta, X_{i}\right), \quad i=1,2 .
$$

Then, given any quasilinear operator $T$ such that

$$
T: M\left(\alpha, X_{1}\right) \rightarrow M\left(\alpha, X_{2}\right) \text { and } T: M\left(\beta, X_{1}\right) \rightarrow M\left(\beta, X_{2}\right),
$$


one has

$$
T: \Gamma_{p, \phi_{1}}\left(X_{1}\right) \rightarrow \Gamma_{p, \phi_{2}}\left(X_{2}\right)
$$

whenever

$$
\begin{gathered}
\int_{0}^{t} s^{\frac{p}{\alpha}-1} \int_{s}^{b} \phi_{2}(y) y^{-\frac{p}{\alpha}} d y d s \leq K \int_{0}^{t} s^{p-1} \int_{s}^{b} \phi_{1}(y) y^{-p} d y d s, \\
t^{\frac{p}{\beta}} \int_{0}^{t} s^{\frac{p}{\beta^{\prime}}-1} \int_{s}^{b} \phi_{2}(y) y^{-p} d y d s \leq K \int_{0}^{t} s^{p-1} \int_{s}^{b} \phi_{1}(y) y^{-p} d y d s,
\end{gathered}
$$

in which $\beta^{\prime}=\frac{\beta}{\beta-1}$ and the constant $K>0$ is independent of $t \in I_{b}$.

In particular, the first condition in (1.4) is necessary and sufficient in order that

$$
S_{\alpha}: \Gamma_{p, \phi_{1}}\left(I_{b}\right) \rightarrow \Gamma_{p, \phi_{2}}\left(I_{b}\right),
$$

while the second one is necessary and sufficient for

$$
T_{\beta}: \Gamma_{p, \phi_{1}}\left(I_{b}\right) \rightarrow \Gamma_{p, \phi_{2}}\left(I_{b}\right) .
$$

The proofs of Theorems A and B appear in Sections 4 and 5, respectively, following the proof of Theorem 1.1 in Section 3. The final section has a number of applications and examples and, as well, a brief discussion of operators on spaces between pairs of the original Lorentz spaces, introduced in [15]. Section 2 to follow outlines the necessary background on r.i. norms and interpolation theory. In particular, it discusses certain r.i. norms whose Boyd and fundamental indices coincide.

\section{Background}

Suppose $(X, \mu)$ is a $\sigma$-finite measure space. Let $\mathfrak{M}(X)=\mathfrak{M}(X, \mu)$ be the class of real-valued $\mu$-measurable functions on $X$. Given $f \in \mathfrak{M}(X)$, we define the decreasing rearrangement, $f^{*}$, of $f$ on $I_{b}:=(0, b), b=$ $\mu(X)$, by

$$
f^{*}(t):=\inf \left\{\lambda>0: \mu_{f}(\lambda) \leq t\right\}, \quad t \in I_{b},
$$

where

$$
\mu_{f}(\lambda):=\mu(\{x \in X:|f(x)|>\lambda\}), \quad \lambda \in \mathbb{R}_{+} .
$$

It satisfies the following inequality of Hardy and Littlewood:

$$
\int_{X}|f(x) g(x)| d \mu(x) \leq \int_{I_{b}} f^{*}(t) g^{*}(t) d t, \quad f, g \in \mathfrak{M}(X) .
$$

The operation of rearrangement is not sublinear though it satisfies

$$
(f+g)^{*}\left(t_{1}+t_{2}\right) \leq f^{*}\left(t_{1}\right)+g^{*}\left(t_{2}\right), \quad f, g \in \mathfrak{M}(X), \quad 0<t_{1}+t_{2}<b .
$$


One does have, however,

$$
(f+g)^{* *}(t) \leq f^{* *}(t)+g^{* *}(t), \quad f, g \in \mathfrak{M}(X), \quad t \in I_{b}
$$

here, the Hardy average, $h^{* *}$, of $h^{*}$, is as defined in the introduction.

Definition 2.1. A rearrangement-invariant (r.i.) Banach function norm, $\bar{\varrho}$, on the class, $\mathfrak{M}\left(I_{b}\right)$, of Lebesgue-measurable functions on $I_{b}$ satisfies the following seven axioms:

(A1) $\bar{\varrho}(f)=\bar{\varrho}(|f|) \geq 0$ with $\bar{\varrho}(f)=0$ if and only if $f=0$ a.e. on $I_{b}$;

(A2) $\bar{\varrho}(c f)=c \bar{\varrho}(f), c \geq 0$;

(A3) $\bar{\varrho}(f+g) \leq \bar{\varrho}(f)+\bar{\varrho}(g)$;

(A4) $f_{n} \uparrow f$ implies $\bar{\varrho}\left(f_{n}\right) \uparrow \bar{\varrho}(f)$;

(A5) $\bar{\varrho}\left(\chi_{E}\right)<\infty$ for all measurable subsets, $E$, of $I_{b}$ with $|E|<\infty$;

(A6) $\int_{E}|f(t)| d t \leq C_{E} \bar{\varrho}(f)$, for all measurable subsets, $E$, of $I_{b}$ with $|E|<\infty$

(A7) $\bar{\varrho}(f)=\bar{\varrho}\left(f^{*}\right)$ or, equivalently, $\mu_{f}=\mu_{g}$ implies $\bar{\varrho}(f)=\bar{\varrho}(g)$.

Using such a $\bar{\varrho}$ one can define an r.i. norm, $\varrho$, on a general $\mathfrak{M}(X)$, with $\mu(X)=b$, by

$$
\varrho(f)=\bar{\varrho}\left(f^{*}\right), \quad f \in \mathfrak{M}(X) .
$$

For details on this and, indeed, all things related to r.i. spaces, we refer to $[\mathbf{1}$, Chapters 1 and 2].

A basic tool for working with r.i. norms $\varrho$ is the Hardy-LittlewoodPólya (HLP) Principle, (see [1, Chapter 2, Theorem 4.6]) which asserts that

$$
f^{* *} \leq g^{* *} \text { implies } \varrho(f) \leq \varrho(g) .
$$

The Köthe dual of an r.i. norm $\varrho$ is another such norm, $\varrho^{\prime}$, with

$$
\varrho^{\prime}(g):=\sup _{\varrho(h) \leq 1} \int_{X}|g(x) h(x)| d \mu(x), \quad g, h \in \mathfrak{M}(X) .
$$

It obeys the Principle of Duality; that is,

$$
\varrho^{\prime \prime}:=\left(\varrho^{\prime}\right)^{\prime}=\varrho .
$$

Further, the Hölder inequality

$$
\int_{X}|f(x) g(x)| d \mu(x) \leq \varrho(f) \varrho^{\prime}(g)
$$

holds for every $f, g \in \mathfrak{M}(X)$. We observe that if $\varrho$ is defined in terms of $\bar{\varrho}$, as in $(2.2)$, then

$$
\varrho^{\prime}(f)=\bar{\varrho}^{\prime}\left(f^{*}\right), \quad f \in \mathfrak{M}(X) .
$$


Corresponding to an r.i. norm $\varrho$ is the set

$$
L_{\varrho}(X):=\{f \in \mathfrak{M}(X): \varrho(f)<\infty\},
$$

which becomes a Banach space with

$$
\|f\|_{L_{\varrho}(X)}:=\varrho(f) ;
$$

indeed, it is a so-called rearrangement-invariant Banach function space or, for short, an r.i. space.

The Orlicz gauge norm is defined in terms of a Young function

$$
A(t):=\int_{0}^{t} a(s) d s, \quad t \geq 0,
$$

in which $a(s)$ is a strictly increasing function on $\mathbb{R}_{+}$, with $a(0+)=0$ and $\lim _{s \rightarrow \infty} a(s)=\infty$. We have

$$
\begin{array}{r}
\varrho_{A}(f):=\inf \left\{\lambda>0: \int_{X} A\left(\frac{|f(x)|}{\lambda}\right) d \mu(x)=\int_{I_{b}} A\left(\frac{f^{*}(t)}{\lambda}\right) d t \leq 1\right\}, \\
f \in \mathfrak{M}(X),
\end{array}
$$

and

$$
L_{A}(X)=L_{\varrho_{A}}(X):=\left\{f \in \mathfrak{M}(X): \varrho_{A}(f)<\infty\right\} .
$$

The Köthe dual of $\varrho_{A}$ is, essentially, the gauge norm $\varrho_{\tilde{A}}$, where

$$
\tilde{A}(t):=\int_{0}^{t} a^{-1}(s) d s, \quad t \in \mathbb{R}_{+},
$$

is called the Young function complementary to $A$; in fact,

$$
\varrho_{\tilde{A}}(g) \leq \varrho_{A}^{\prime}(g) \leq 2 \varrho_{\tilde{A}}(g), \quad g \in \mathfrak{M}(X) .
$$

Given an index $p, 1<p<\infty$, and a nontrivial weight $\phi$ on $I_{b}$, the Lorentz gamma norm, $\varrho_{p, \phi}$, is defined by

$$
\varrho_{p, \phi}(f):=\left[\int_{I_{b}} f^{* *}(t)^{p} \phi(t) d t\right]^{\frac{1}{p}}, \quad f \in \mathfrak{M}(X) .
$$

This norm determines the Lorentz gamma space

$$
\Gamma_{p, \phi}(X)=L_{\varrho_{p, \phi}}:=\left\{f \in \mathfrak{M}(X): \varrho_{p, \phi}(f)<\infty\right\} .
$$

As mentioned in the introduction, we require

$$
\int_{1}^{\infty} \phi(t) t^{-p} d t<\infty \text {, when } b=\infty \text {, and } \int_{I_{b}} \phi(t) t^{-p} d t=\infty \text {, when } b<\infty \text {. }
$$


The Köthe dual of $\varrho_{p, \phi}$ is equivalent to the Lorentz gamma norm $\varrho_{p^{\prime}, \psi}$, with $p^{\prime}=\frac{p}{p-1}$ and

$$
\psi(t):=\frac{t^{p^{\prime}+p-1} \int_{0}^{t} \phi(s) d s \int_{t}^{b} \phi(s) s^{-p} d s}{\left(\int_{0}^{t} \phi(s) d s+t^{p} \int_{t}^{b} \phi(s) s^{-p} d s\right)^{p^{\prime}+1}}, \quad t \in I_{b},
$$

provided

$$
\int_{0}^{1} \phi(t) t^{-p} d t=\int_{1}^{\infty} \phi(t) d t=\infty, \quad \text { if } b=\infty .
$$

See [10, Theorem 6.2].

The dilation operator, $E_{s}, s \in \mathbb{R}_{+}$, given at $f \in \mathfrak{M}\left(I_{b}\right), 0<b \leq \infty$, and $t \in I_{b}$, by

$$
\left(E_{s} f\right)(t):= \begin{cases}f(t / s), & \text { if } 0<t<b s, \\ 0, & \text { if } b s \leq t<b,\end{cases}
$$

is bounded on any r.i. space $L_{\bar{\varrho}}\left(I_{b}\right)$ [1, Chapter 3, Proposition 5.11]. Denote the norm of $E_{s}$ on $L_{\bar{\rho}}\left(I_{b}\right)$ by $h_{\bar{\rho}}(s)$ and define the lower and upper Boyd indices of $L_{\bar{\varrho}}\left(I_{b}\right)$ as

$$
i_{\bar{\varrho}}:=\lim _{s \rightarrow \infty} \frac{\log s}{\log h_{\bar{\varrho}}(s)} \text { and } I_{\bar{\varrho}}:=\lim _{s \rightarrow 0+} \frac{\log s}{\log h_{\bar{\varrho}}(s)},
$$

respectively. They satisfy

$$
1 \leq i_{\bar{\varrho}} \leq I_{\bar{\varrho}} \leq \infty
$$

also

$$
i_{\bar{\varrho}^{\prime}}=\frac{I_{\bar{\varrho}}}{I_{\bar{\varrho}}-1} \text { and } I_{\bar{\varrho}^{\prime}}=\frac{i_{\bar{\varrho}}}{i_{\bar{\varrho}}-1} .
$$

See [14, Vol. II, pp. 131-132].

If in (2.4) we replace $h_{\bar{\varrho}}(s)$ by the norm, $k_{\bar{\varrho}}(s)$ of $E_{s}$ on characteristic functions of sets of finite measure, we obtain the so-called fundamental indices.

The following result is proved in $[\mathbf{3}]$.

Theorem 2.2. Fix $\alpha, \beta$ and $b$ with $1<\alpha<\beta<\infty$ and $0<b \leq \infty$. Set $\left(P_{\alpha} f\right)(t):=t^{-\frac{1}{\alpha}} \int_{0}^{t} f(s) s^{\frac{1}{\alpha}-1} d s$ and $\left(Q_{\beta} f\right)(t):=t^{-\frac{1}{\beta}} \int_{t}^{b} f(s) s^{\frac{1}{\beta}-1} d s$ for suitable $f \in \mathfrak{M}\left(I_{b}\right)$ and $t \in I_{b}$. Let $\underline{\varrho}$ be an r.i. norm on $\mathfrak{M}\left(I_{b}\right)$. Then,

$$
P_{\alpha}: L_{\bar{\varrho}}\left(I_{b}\right) \rightarrow L_{\bar{\varrho}}\left(I_{b}\right) \text { if and only if } i_{\bar{\varrho}}>\alpha ;
$$

again,

$$
Q_{\beta}: L_{\bar{\varrho}}\left(I_{b}\right) \rightarrow L_{\bar{\varrho}}\left(I_{b}\right) \text { if and only if } I_{\bar{\varrho}}<\beta .
$$


In case $\bar{\varrho}=\varrho_{A}$ is an Orlicz norm, one has

$$
h_{\varrho}(s) \approx \lim _{t \rightarrow 0+} \frac{A^{-1}(1 / t)}{A^{-1}(1 / s t)} .
$$

This reflects the fact that the norm of $E_{s}$ on an Orlicz space is essentially determined on characteristic functions of sets of finite measure and that $\varrho_{A}\left(\chi_{E}\right)=\frac{1}{A^{-1}\left(|E|^{-1}\right)}$. The same is true for Lorentz gamma spaces. This is the content of the following result from $[\mathbf{8}]$.

Theorem 2.3. Let $(X, \mu)$ be a $\sigma$-finite measure space with $\mu(X)=b$. Fix an index $p, 1<p<\infty$, and suppose $\phi$ is a nontrivial weight function on $I_{b}$. Take $\bar{\varrho}(f)=\varrho_{p, \phi}(f), f \in \mathfrak{M}\left(I_{b}\right)$. Set

$$
h_{\bar{\varrho}}=\sup \frac{\bar{\varrho}\left(E_{t} f\right)}{\bar{\varrho}(f)}, \quad t \in \mathbb{R}_{+}, 0 \neq f \in \mathfrak{M}\left(I_{b}\right),
$$

and define the Boyd indices $i_{\bar{\varrho}}$ and $I_{\bar{\varrho}}$ as in (2.4). Then, these indices can be computed by using the formula

$$
h_{\bar{\varrho}}(s) \approx \sup _{0<t<b}\left[\frac{\int_{0}^{s t} \phi(y) d y+s^{p} t^{p} \int_{s t}^{b} \phi(y) y^{-p} d y}{\int_{0}^{t} \phi(y) d y+t^{p} \int_{t}^{b} \phi(y) y^{-p} d y}\right]^{\frac{1}{p}} .
$$

We now describe certain parts of Interpolation Theory used later on.

Let $\left(X_{0}, X_{1}\right)$ be a pair of Banach spaces compatible in the sense that they are continuously imbedded in a common Hausdorff topological vector space $H$. Their $K$-functional is defined for each $f$ in the vector sum $X_{0}+X_{1}$ by

$$
K\left(t, f ; X_{0}, X_{1}\right):=\inf _{f=g+h}\left[\|g\|\left\|_{X_{0}}+t\right\| h \|_{X_{1}}\right], \quad t \in \mathbb{R}_{+} .
$$

The $K$-functional is a nonnegative, increasing, concave function of $t$ on $\mathbb{R}_{+}$; see [1, Proposition 2, p. 294]. So,

$$
K\left(t, f ; X_{0}, X_{1}\right)=K\left(0+, f ; X_{0}, X_{1}\right)+\int_{0}^{t} k\left(s, f ; X_{0}, X_{1}\right) d s, \quad t \in \mathbb{R}_{+},
$$

in which the $k$-functional, $k\left(t, f ; X_{0}, X_{1}\right)$, is a uniquely defined nonnegative, right-continuous, decreasing function on $\mathbb{R}_{+}$. According to $[\mathbf{1}$, Proposition 1.15, p. 303],

$$
K\left(0+, f ; X_{0}, X_{1}\right)=0 \text { for all } f \in X_{0}+X_{1}
$$

if and only if $X_{0} \cap X_{1}$ is dense in $X_{0}$. 
Next, we restrict attention to r.i. spaces of functions in the context of a $\sigma$-finite measure space $(X, \mu)$, with $\mu(X)=b$. Such spaces are continuously imbedded in the Hausdorff topological vector space consisting of the set $\mathfrak{M}(X)$ together with the (metrizable) topology of convergence on sets of finite measure.

A special case of $[\mathbf{1}$, Theorem 1.19 , pp. $305-306]$ is

Theorem 2.4. Let $\varrho_{0}, \varrho_{1}, \sigma_{0}$ and $\sigma_{1}$ be r.i. norms on $\mathfrak{M}(X)$ defined in terms of the norms $\bar{\varrho}_{0}, \bar{\varrho}_{1}, \bar{\sigma}_{0}$ and $\bar{\sigma}_{1}$ on $\mathfrak{M}\left(I_{b}\right)$. Given the r.i. norm $\lambda$ on $\mathfrak{M}\left(\mathbb{R}_{+}\right), g \in \mathfrak{M}\left(I_{b}\right)$ and $f \in \mathfrak{M}(X)$, set

$$
\bar{\varrho}(g):=\lambda\left(k\left(t, g ; L_{\varrho_{0}}\left(I_{b}\right), L_{\bar{\varrho}_{1}}\left(I_{b}\right)\right)\right)
$$

and

$$
\bar{\sigma}(g):=\lambda\left(k\left(t, g ; L_{\bar{\sigma}_{0}}\left(I_{b}\right), L_{\bar{\sigma}_{1}}\left(I_{b}\right)\right)\right)
$$

also

$$
\varrho(f):=\bar{\varrho}\left(f^{*}\right) \text { and } \sigma(f):=\bar{\sigma}\left(f^{*}\right) .
$$

Then, $L_{\varrho}=L_{\varrho}(X)$ and $L_{\sigma}=L_{\sigma}(X)$ are r.i. spaces of functions in $\mathfrak{M}(X)$ with the norms $\|f\|_{\varrho}:=\varrho(f)$ and $\|f\|_{\sigma}:=\sigma(f)$. Moreover, if $T$ is any linear operator on $L_{\varrho_{0}}+L_{\varrho_{1}}$ satisfying

$$
T: L_{\varrho_{0}} \rightarrow L_{\sigma_{0}} \text { and } T: L_{\varrho_{1}} \rightarrow L_{\sigma_{1}}
$$

then, $T: L_{\varrho} \rightarrow L_{\sigma}$. In particular, $L_{\varrho}$ is an interpolation space between $L_{\varrho_{0}}$ and $L_{\varrho_{1}}$ in the sense that, for any linear operator $T$,

$$
T: L_{\varrho_{0}} \rightarrow L_{\varrho_{0}} \text { and } T: L_{\varrho_{1}} \rightarrow L_{\varrho_{1}},
$$

implies $T: L_{\varrho} \rightarrow L_{\varrho}$; similarly, $L_{\sigma}$ is an interpolation space between $L_{\sigma_{0}}$ and $L_{\sigma_{1}}$.

Lastly, we recall that, for $1<p \leq \infty, 1 \leq q \leq \infty$, the Lorentz norms, $\varrho_{p, q}$, are defined at $f \in \mathfrak{M}(X), \mu(X)=b$, by

$$
\varrho_{p q}(f):=\left(\int_{I_{b}}\left[t^{\frac{1}{p}-\frac{1}{q}} f^{* *}(t)\right]^{q} d t\right)^{\frac{1}{q}}, \text { when } q<\infty,
$$

and

$$
\varrho_{p \infty}(f):=\sup _{0<t<b} t^{\frac{1}{p}} f^{* *}(t) ;
$$

see $[\mathbf{1 1}]$. We will write $L_{\varrho_{p q}}(X)$ as $\Lambda(p, q, X)$, using the special notation $\Lambda(p, X)$ when $q=1$ and $M(p, X)$ when $q=\infty$. 


\section{The proof of Theorem 1.1}

Proof: Given (1.2), the fundamental result on $K$-functionals [1, Chapter 5, Theorem 1.11, p. 301] and the Holmstedt formula [1, Chapter 5, Theorem 2.1, pp. 307-309]

$$
K\left(t, g ; M\left(\alpha, X_{i}\right), M\left(\beta, X_{i}\right)\right) \approx \sup _{0<s \leq t^{\gamma}} s^{\frac{1}{\alpha}} g^{*}(s)+t \sup _{t^{\gamma} \leq s<b} s^{\frac{1}{\beta}} g^{*}(s),
$$

where $g \in M\left(\alpha, X_{i}\right)+M\left(\beta, X_{i}\right), i=1,2$ and $\frac{1}{\gamma}=\frac{1}{\alpha}-\frac{1}{\beta}$, one has

$$
\begin{aligned}
\sup _{0<s \leq t} s^{\frac{1}{\alpha}}(T f)^{*}(s)+t^{\frac{1}{\gamma}} & \sup _{t \leq s<b} s^{\frac{1}{\beta}}(T f)^{*}(s) \\
& \leq C \sup _{0<s \leq C t} s^{\frac{1}{\alpha}} f^{*}(s)+C t^{\frac{1}{\gamma}} \sup _{C t \leq s<b} s^{\frac{1}{\beta}} f^{*}(s),
\end{aligned}
$$

with $C>1$ independent of $t, 0<t<\frac{b}{C}$. Hence, by [12, (3.19)],

$$
\begin{aligned}
\sup _{0<s \leq t} s^{\frac{1}{\alpha}}(T f)^{* *}(s) \approx \sup _{0<s \leq t} s^{\frac{1}{\alpha}}(T f)^{*}(s) & \\
& \leq C \sup _{0<s \leq C t} s^{\frac{1}{\alpha}} f^{*}(s)+C t^{\frac{1}{\gamma}} \sup _{C t \leq s<b} s^{\frac{1}{\beta}} f^{*}(s)
\end{aligned}
$$

and so, for some $K>C$,

$$
t^{\frac{1}{\alpha}}(T f)^{* *}(t) \leq K \sup _{0<s \leq C t} s^{\frac{1}{\alpha}} f^{*}(s)+K t^{\frac{1}{\gamma}} \sup _{C t \leq s<b} s^{\frac{1}{\beta}} f^{*}(s), \quad 0<t<\frac{b}{C} .
$$

Dividing both sides by $t^{\frac{1}{\alpha}}$, we arrive at

$$
(T f)^{* *}(t) \leq K^{2}\left(S_{\alpha} f+T_{\beta} f\right)(C t) \leq K^{2}\left(S_{\alpha} f+T_{\beta} f\right)^{* *}(C t), \quad 0<t<\frac{b}{C} .
$$

From this, HLP, (1.2) and the continuity of the dilation operator yield $\varrho_{2}(T f)=\bar{\varrho}_{2}\left((T f)^{*}\right) \leq K^{2} \bar{\varrho}_{2}\left(\left(S_{\alpha} f+T_{\beta} f\right)^{*}(C t)\right) \leq M \bar{\varrho}_{1}\left(f^{*}\right)=M \varrho_{1}(f)$,

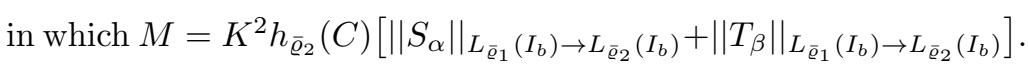

\section{The proof of Theorem A}

Lemma 4.1. Fix $\alpha>1$ and $b \in(0, \infty]$. Let $A$ be a Young function satisfying $t^{-\frac{1}{\alpha}} \notin L_{A}\left(I_{b}\right)$,

$$
A(t)=0, \quad t \in I_{b^{-1}},
$$

when $b<\infty$, and

$$
\int_{0}^{1} A(t) t^{-1-\alpha} d t<\infty
$$


when $b=\infty$. Then,

$$
E_{\alpha}(t):=\alpha t^{\alpha} \int_{b^{-1}}^{t} \frac{A(s)}{s^{\alpha+1}} d s
$$

is a strictly increasing function of $t$ on $\left(b^{-1}, \infty\right)$, with

$$
\varrho_{A}\left(s^{-\frac{1}{\alpha}} \chi_{(t, b)}(s)\right)=\frac{t^{-\frac{1}{\alpha}}}{E_{\alpha}^{-1}\left(t^{-1}\right)}
$$

for all $t \in \mathbb{R}_{+}$when $b=\infty$ and for sufficiently small $t$ when $b<\infty$.

Proof: This is essentially a modification of (4.44) in [5, p. 63]. We deal only with the case $b<\infty$, the proof being, in fact, simpler when $b=\infty$.

Now, $\varrho_{A}\left(s^{-\frac{1}{\alpha}} \chi_{(t, b)}(s)\right)$ is, by definition, the number $\lambda$ such that

$$
\int_{t}^{b} A\left(\frac{s^{-\frac{1}{\alpha}}}{\lambda}\right) d s=1
$$

or, with $y=\frac{s^{-\frac{1}{\alpha}}}{\lambda}$

$$
\frac{\alpha}{\lambda^{\alpha}} \int_{\max \left\{b^{-1}, b^{-\frac{1}{\alpha}} \lambda^{-1}\right\}}^{t^{-\frac{1}{\alpha}} \lambda^{-1}} \frac{A(y)}{y^{\alpha+1}} d y=1 .
$$

Since $t^{-\frac{1}{\alpha}} \notin L_{A}\left(I_{b}\right)$, one has $\lim _{t \rightarrow 0_{+}} \varrho_{A}\left(s^{-\frac{1}{\alpha}} \chi_{(t, b)}(s)\right)=\infty$. Hence, for sufficiently small $t$, we obtain $b^{-\frac{1}{\alpha}} \lambda^{-1} \leq b^{-1}$, and (4.2) becomes

$$
\frac{\alpha}{\lambda^{\alpha}} \int_{b^{-1}}^{t^{-\frac{1}{\alpha}} \lambda^{-1}} \frac{A(y)}{y^{\alpha+1}} d y=1
$$

that is,

$$
E_{\alpha}\left(\frac{1}{\lambda t^{\frac{1}{\alpha}}}\right)=t^{-1}
$$

Thus,

or

$$
\frac{1}{\lambda t^{\frac{1}{\alpha}}}=E_{\alpha}^{-1}\left(t^{-1}\right),
$$

$$
\lambda=\frac{t^{-\frac{1}{\alpha}}}{E_{\alpha}^{-1}\left(t^{-1}\right)} .
$$

Lemma 4.2. Fix $\beta>1$ and let $A$ be a Young function satisfying

$$
\int_{1}^{\infty} A(t) t^{-1-\beta} d t<\infty \text {. }
$$

Then,

$$
F_{\beta}(t):=\beta t^{\beta} \int_{t}^{\infty} \frac{A(s)}{s^{\beta+1}} d s
$$


is a strictly increasing function of $t$ on $\mathbb{R}_{+}$, with

$$
\varrho_{A}\left(s^{-\frac{1}{\beta}} \chi_{(0, t)}(s)\right)=\frac{t^{-\frac{1}{\beta}}}{F_{\beta}^{-1}\left(t^{-1}\right)}, \quad t \in \mathbb{R}_{+} .
$$

Proof: Similar to that of Lemma 4.1.

Proof of Theorem A: Theorem 1.1 guarantees

$$
T: L_{A_{1}}\left(X_{1}\right) \rightarrow L_{A_{2}}\left(X_{2}\right)
$$

whenever

$$
S_{\alpha}: L_{A_{1}}\left(I_{b}\right) \rightarrow L_{A_{2}}\left(I_{b}\right) \text { and } T_{\beta}: L_{A_{1}}\left(I_{b}\right) \rightarrow L_{A_{2}}\left(I_{b}\right) .
$$

We will prove the equivalence of the boundedness of $S_{\alpha}$ and the first of the conditions in (1.3), namely,

$$
\int_{b^{-1}}^{t} \frac{A_{2}(s)}{s^{\alpha+1}} d s \leq \frac{A_{1}(K t)}{t^{\alpha}}, \quad t>b^{-1} .
$$

The proof that the boundedness of $T_{\beta}$ is equivalent to the second condition in (1.3) is similar.

To begin, assume

$$
S_{\alpha}: L_{A_{1}}\left(I_{b}\right) \rightarrow L_{A_{2}}\left(I_{b}\right)
$$

and let $t \in I_{b}$. A simple calculation shows

$$
\left(S_{\alpha} \chi_{(0, t)}\right)(s)=\chi_{(0, t)}(s)+t^{\frac{1}{\alpha}} s^{-\frac{1}{\alpha}} \chi_{(t, b)}(s), \quad s \in I_{b} .
$$

Therefore,

$$
\varrho_{A_{2}}\left(S_{\alpha} \chi_{(0, t)}\right) \geq t^{\frac{1}{\alpha}} \varrho_{A_{2}}\left(s^{-\frac{1}{\alpha}} \chi_{(t, b)}(s)\right), \quad t \in I_{b},
$$

so, with $f=\chi_{(0, t)},(4.4)$ ensures

$$
t^{\frac{1}{\alpha}} \varrho_{A_{2}}\left(s^{-\frac{1}{\alpha}} \chi_{(t, b)}(s)\right) \leq C \varrho_{A_{1}}\left(\chi_{(0, t)}\right)=\frac{C}{A_{1}^{-1}\left(t^{-1}\right)},
$$

$C>0$ being independent of $t \in I_{b}$. In view of Lemma 4.1, (4.5) implies

$$
\frac{1}{E_{\alpha}^{-1}\left(t^{-1}\right)} \leq \frac{C}{A_{1}^{-1}\left(t^{-1}\right)}
$$

for sufficiently small $t$, in which $E_{\alpha}$ is defined with respect to $A_{2}$. Since $E_{\alpha}$ is increasing, we conclude that there exists some $t_{0} \geq b^{-1}$ such that

$$
E_{\alpha}(t) \leq \alpha^{-1} A_{1}(C t), \quad t \geq t_{0} .
$$


Since $\alpha>1$, this yields

$$
E_{\alpha}(t) \leq A_{1}(C t), \quad t \geq t_{0}
$$

Setting

$$
C^{\prime}:=\sup _{t \in\left[b^{-1}, t_{0}\right]} \frac{A_{1}^{-1}\left(E_{\alpha}(t)\right)}{t}
$$

and

$$
K:=\max \left\{C, C^{\prime}\right\}
$$

we get (4.3).

Suppose now that (4.3) holds. Fix $0 \leq f \in L_{A_{1}}\left(X_{1}\right), \varrho_{A_{1}}(f)=1$, and, for $t \in \mathbb{R}_{+}$, define

$$
f_{t}(s)=\min \left[f^{*}(s), t\right] \text { and } f^{t}(s)=f^{*}(s)-f_{t}(s), \quad s \in I_{b} .
$$

Then, $f^{t}$ and $f_{t}$ are nonnegative and decreasing,

$$
\left(S_{\alpha} f_{t}\right)(s) \leq t, \quad s \in I_{b}
$$

and, since, by (2.1),

$$
f^{*}(2 s)=\left(f_{t}+f^{t}\right)(2 s) \leq f_{t}(s)+f^{t}(s), \quad 0<s<\frac{b}{2},
$$

we have

$$
\left(S_{\alpha} f\right)(2 s) \leq\left(S_{\alpha} f_{t}\right)(s)+\left(S_{\alpha} f^{t}\right)(s), \quad 0<s<\frac{b}{2}
$$

We observe that, by the argument of [12, Lemma 3.5], one has (4.8) $t^{\alpha}\left|\left\{S_{\alpha} g>t\right\}\right| \leq C \sup _{s \in \mathbb{R}_{+}} s^{\alpha}|\{|g| g>s\}|, \quad g \in \mathfrak{M}\left(I_{b}\right), \quad t \in \mathbb{R}_{+}$. 
Thus, with $A_{2}^{\prime}=a_{2}$,

$$
\begin{aligned}
& \int_{0}^{\frac{b}{2}} A_{2}\left(\frac{1}{2}\left(S_{\alpha} f\right)(2 t)\right) d t \\
= & \int_{\mathbb{R}_{+}} a_{2}(t)\left|\left\{s \in\left(0, \frac{b}{2}\right):\left(S_{\alpha} f\right)(2 s)>2 t\right\}\right| d t \\
\leq & \int_{\mathbb{R}_{+}} a_{2}(t)\left|\left\{s \in\left(0, \frac{b}{2}\right):\left(S_{\alpha} f_{t}\right)(s)>t\right\}\right| d t \\
& +\int_{\mathbb{R}_{+}} a_{2}(t)\left|\left\{s \in\left(0, \frac{b}{2}\right):\left(S_{\alpha} f^{t}\right)(s)>t\right\}\right| d t, \quad \text { by (4.7), } \\
= & \int_{\mathbb{R}_{+}} a_{2}(t)\left|\left\{s \in\left(0, \frac{b}{2}\right):\left(S_{\alpha} f^{t}\right)(s)>t\right\}\right| d t, \quad \text { by (4.6), } \\
\leq & C \int_{\mathbb{R}_{+}} a_{2}(t) t^{-\alpha} \sup _{s \in \mathbb{R}_{+}} s^{\alpha}\left|\left\{y \in I_{b}: f^{t}(y)>s\right\}\right| d t, \quad \text { by (4.8), } \\
= & C \int_{\mathbb{R}_{+}} a_{2}(t) t^{-\alpha} \sup _{s \geq t}(s-t)^{\alpha}\left|\left\{y \in I_{b}: f^{*}(y)>s\right\}\right| d t \\
\leq & C \int_{0}^{b^{-1}} a_{2}(t) t^{-\alpha} \sup _{s \geq t} s^{\alpha}\left|\left\{y \in I_{b}: f^{*}(y)>s\right\}\right| d t \\
& +C \int_{b^{-1}}^{\infty} a_{2}(t) t^{-\alpha} \sup _{s \geq t} s^{\alpha}\left|\left\{y \in I_{b}: f^{*}(y)>s\right\}\right| d t .
\end{aligned}
$$

Now, the first term is no bigger than

$$
C \varrho_{M\left(\alpha, X_{1}\right)}(f) \int_{0}^{b^{-1}} a_{2}(t) t^{-\alpha} d t
$$

which, in turn, using the inequality $t a_{2}(t) \leq A_{2}(2 t)$, is majorized by

$$
C \varrho_{M\left(\alpha, X_{1}\right)}(f) \int_{0}^{b^{-1}} A_{2}(2 t) t^{-\alpha-1} d t=2^{\alpha} C \varrho_{M\left(\alpha, X_{1}\right)}(f) \int_{0}^{2 b^{-1}} A_{2}(t) t^{-\alpha-1} d t,
$$

this being finite by assumption. We observe that, if $b<\infty$, one has

$$
M\left(\alpha, X_{i}\right)+M\left(\beta, X_{i}\right)=M\left(\alpha, X_{i}\right), \quad i=1,2,
$$

while, if $b=\infty$, the first term is zero.

For the second term we have

$C \int_{b^{-1}}^{\infty} a_{2}(t) t^{-\alpha} \sup _{s \geq t} s^{\alpha}\left|\left\{y \in I_{b}: f^{*}(y)>s\right\}\right| d t \leq C \int_{b^{-1}}^{\infty} a_{2}(t)\left(T_{\frac{1}{\alpha}} h\right)(t) d t$,

where

$$
h(t):=\left|\left\{y \in I_{b}: f^{*}(y)>t\right\}\right|
$$


and

$$
\left(T_{\frac{1}{\alpha}} h\right)(t):=t^{-\alpha} \sup _{s \geq t} s^{\alpha} h(s), \quad t>b^{-1} .
$$

As $a_{2}(s) \leq s^{-1} A_{2}(2 s),(4.3)$ implies

$$
t^{\alpha} \int_{b^{-1}}^{t} \frac{a_{2}(s)}{s^{\alpha}} d s \leq A_{1}(2 K t), \quad t>b^{-1} .
$$

A slight modification of [9, Theorem 3.2] guarantees there exists a $K>0$ such that, with $A_{1}^{\prime}=a_{1}$,

$$
\begin{array}{r}
\int_{b^{-1}}^{\infty} a_{2}(t)\left(T_{\frac{1}{\alpha}} h\right)(t) d t \leq \int_{b^{-1}}^{\infty} a_{1}(K t) h(t) d t \leq \int_{I_{b}} A_{1}(K f(t)) d t, \\
0 \leq f \in \mathfrak{M}\left(I_{b}\right) .
\end{array}
$$

Altogether, then,

$$
\begin{aligned}
\int_{0}^{\frac{b}{2}} A_{2}\left(\frac{1}{2}\left(S_{\alpha} f\right)(2 t)\right) d t \leq 2^{\alpha} C \varrho_{M\left(\alpha, X_{1}\right)}(f) & \int_{0}^{2 b^{-1}} A_{2}(t) t^{-\alpha-1} d t \\
& +C \int_{I_{b}} A_{1}(K f(t)) d t,
\end{aligned}
$$

or

$$
\begin{aligned}
\int_{I_{b}} A_{2}\left(\frac{1}{2}\left(S_{\alpha} f\right)(t)\right) d t \leq 2^{\alpha+1} C \varrho_{M\left(\alpha, X_{1}\right)}(f) & \int_{0}^{2 b^{-1}} A_{2}(t) t^{-\alpha-1} d t \\
& +2 C \int_{I_{b}} A_{1}(K f(t)) d t,
\end{aligned}
$$

$0 \leq f \in \mathfrak{M}\left(I_{b}\right)$, from which (4.4) follows by a standard argument.

\section{The proof of Theorem B}

Proof of Theorem B: We proceed as in the proof of Theorem A. Thus,

$$
T: \Gamma_{p, \phi_{1}}\left(X_{1}\right) \rightarrow \Gamma_{p, \phi_{2}}\left(X_{2}\right)
$$

follows from

$$
S_{\alpha}: \Gamma_{p, \phi_{1}}\left(I_{b}\right) \rightarrow \Gamma_{p, \phi_{2}}\left(I_{b}\right) \text { and } T_{\beta}: \Gamma_{p, \phi_{1}}\left(I_{b}\right) \rightarrow \Gamma_{p, \phi_{2}}\left(I_{b}\right) .
$$

The connection of the latter to (1.4) will be achieved by our showing

$$
S_{\alpha}: \Gamma_{p, \phi_{1}}\left(I_{b}\right) \rightarrow \Gamma_{p, \phi_{2}}\left(I_{b}\right)
$$

if and only if

$$
\int_{0}^{t} s^{\frac{p}{\alpha}-1} \int_{s}^{b} \phi_{2}(y) y^{-\frac{p}{\alpha}} d y d s \leq K \int_{0}^{t} s^{p-1} \int_{s}^{b} \phi_{1}(y) y^{-p} d y d s, \quad t \in I_{b},
$$


and

$$
T_{\beta}: \Gamma_{p, \phi_{1}}\left(I_{b}\right) \rightarrow \Gamma_{p, \phi_{2}}\left(I_{b}\right)
$$

if and only if

$$
t^{\frac{p}{\beta}} \int_{0}^{t} s^{\frac{p}{\beta^{\prime}}-1} \int_{s}^{b} \phi_{2}(y) y^{-p} d y d s \leq K \int_{0}^{t} s^{p-1} \int_{s}^{b} \phi_{1}(y) y^{-p} d y d s, \quad t \in I_{b} .
$$

We observe that for $f \in M\left(I_{b}\right), t \in I_{b}$, one has

$$
S_{\alpha} f(t)=\sup _{0<y \leq 1} y^{\frac{1}{\alpha}} f^{*}(t y),
$$

whence $S_{\alpha} f$ is nonincreasing on $I_{b}$, that is, $S_{\alpha} f=\left(S_{\alpha} f\right)^{*}$. Thus, $\left(S_{\alpha} f\right)(t) \leq\left(S_{\alpha} f\right)^{* *}(t), t \in I_{b}$. Using this and [12, Theorem 3.6], we conclude that (5.1) is equivalent to

$$
\int_{I_{b}}\left(S_{\alpha} f\right)(s)^{p} \phi_{2}(s) d s \leq C \int_{I_{b}} f^{* *}(s)^{p} \phi_{1}(s) d s, \quad f \in \mathfrak{M}_{+}\left(I_{b}\right) .
$$

Taking $f=\chi_{(0, t)}$, this reads

$$
\int_{I_{b}}\left(S_{\alpha} \chi(0, t)\right)(s)^{p} \phi_{2}(s) d s \leq C \int_{0}^{t} \chi_{(0, t)}^{* *}(s)^{p} \phi_{1}(s) d s, \quad t \in I_{b} .
$$

But,

$$
\begin{aligned}
\int_{I_{b}}\left(S_{\alpha} \chi_{(0, t)}\right)(s)^{p} \phi_{2}(s) d s & =\int_{0}^{t} \phi_{2}(s) d s+t^{\frac{p}{\alpha}} \int_{t}^{b} \phi_{2}(s) s^{-\frac{p}{\alpha}} d s \\
& =\frac{p}{\alpha} \int_{0}^{t} s^{\frac{p}{\alpha}-1} \int_{s}^{b} \phi_{2}(y) y^{-\frac{p}{\alpha}} d y, \quad t \in I_{b} .
\end{aligned}
$$

Further,

$$
\chi_{(0, t)}^{* *}(s)=\min \left[1, \frac{t}{s}\right]
$$

so,

$$
\begin{aligned}
\int_{I_{b}} \chi_{(0, t)}^{* *}(s)^{p} \phi_{1}(s) d s & =\int_{0}^{t} \phi_{1}(s) d s+t^{p} \int_{t}^{b} \phi_{1}(s) s^{-p} d s \\
& =p \int_{0}^{t} s^{p-1} \int_{s}^{b} \phi_{1}(y) y^{-p} d y d s .
\end{aligned}
$$

Therefore, when (5.1) holds, we get (5.2) with $K=C \alpha$.

Suppose, next, that (5.2) holds. We claim

$$
\left(S_{\alpha} f(s)\right)^{p} \leq \frac{p}{\alpha} s^{-\frac{p}{\alpha}} \int_{0}^{s} f^{*}(y)^{p} y^{\frac{p}{\alpha}-1} d y, \quad f \in \mathfrak{M}_{+}\left(I_{b}\right), s \in I_{b} .
$$


Indeed, for each $z, 0<z \leq s$,

$\int_{0}^{s} f^{*}(y)^{p} y^{\frac{p}{\alpha}-1} d y \geq \int_{0}^{z} f^{*}(y)^{p} y^{\frac{p}{\alpha}-1} d y \geq f^{*}(z)^{p} \int_{0}^{z} y^{\frac{p}{\alpha}-1} d y=\frac{\alpha}{p} z^{\frac{p}{\alpha}} f^{*}(z)^{p}$,

and hence

$$
\frac{p}{\alpha} s^{-\frac{p}{\alpha}} \int_{0}^{s} f^{*}(y)^{p} y^{\frac{p}{\alpha}-1} d y \geq s^{-\frac{p}{\alpha}} \sup _{0<z \leq s} z^{\frac{p}{\alpha}} f^{*}(z)^{p}=\left(S_{\alpha} f\right)(s)^{p} .
$$

Thus, (5.1) will follow once we show

$$
\int_{I_{b}} s^{-\frac{p}{\alpha}} \int_{0}^{s} f^{*}(y)^{p} y^{\frac{p}{\alpha}-1} d y \phi_{2}(s) d s \leq C \int_{I_{b}} f^{* *}(s)^{p} \phi_{1}(s) d s, \quad f \in \mathfrak{M}_{+}\left(I_{b}\right) .
$$

Interchanging the order of integration on the left hand side this becomes

$$
\int_{I_{b}} f^{*}(y)^{p} y^{\frac{p}{\alpha}-1} \int_{y}^{b} s^{-\frac{p}{\alpha}} \phi_{2}(s) d s \leq C \int_{I_{b}} f^{* *}(s)^{p} \phi_{1}(s) d s, \quad f \in \mathfrak{M}_{+}\left(I_{b}\right) .
$$

According to [17, Theorem 3.2],

$$
\int_{I_{b}} f^{*}(y)^{p} y^{p-1} \int_{y}^{b} \phi_{1}(s) s^{-p} d s d y \leq C \int_{I_{b}} f^{* *}(s)^{p} \phi_{1}(s) d s,
$$

so (5.5) would be a consequence of

$$
\begin{array}{r}
\int_{I_{b}} f^{*}(y)^{p} y^{\frac{p}{\alpha}-1} \int_{y}^{b} \phi_{2}(s) s^{-\frac{p}{\alpha}} d s d y \leq C \int_{I_{b}} f^{*}(y)^{p} y^{p-1} \int_{y}^{b} \phi_{1}(s) s^{-p} d s d y, \\
f \in \mathfrak{M}_{+}\left(I_{b}\right) .
\end{array}
$$

Finally, [18, Remark (i), p. 148] asserts that this last inequality holds if and only if (5.2) is satisfied.

It remains to prove the equivalence of (5.3) and (5.4). To begin, (5.3) ensures that, for every $t \in I_{b}$,

$$
\begin{aligned}
\int_{I_{b}}\left(T_{\beta} \chi_{(0, t)}\right)^{* *}(s)^{p} \phi_{2}(s) d s & \leq C \int_{I_{b}} \chi_{(0, t)}^{* *}(s)^{p} \phi_{1}(s) d s \\
& =C p \int_{0}^{t} s^{p-1} \int_{s}^{b} \phi_{1}(y) y^{-p} d y d s .
\end{aligned}
$$

But,

$$
\left(T_{\beta} \chi_{(0, t)}\right)(s)=\left(\frac{t}{s}\right)^{\frac{1}{\beta}} \chi_{(0, t)}(s), \quad t \in I_{b},
$$


so,

$$
\begin{aligned}
\left(T_{\beta} \chi_{(0, t)}\right)^{* *}(s) & =s^{-1} \int_{0}^{s}\left(\frac{t}{s}\right)^{\frac{1}{\beta}} d y \chi_{(0, t)}(s)+s^{-1} \int_{0}^{s}\left(\frac{t}{y}\right)^{\frac{1}{\beta}} d y \chi_{(t, b)}(s) \\
& =\frac{\beta}{\beta-1}\left[\left(\frac{t}{s}\right)^{\frac{1}{\beta}} \chi_{(0, t)}(s)+\frac{t}{s} \chi_{(t, b)}(s)\right]
\end{aligned}
$$

and, therefore,

$$
\begin{aligned}
\int_{I_{b}}\left(T_{\beta} \chi(0, t)\right)^{* *}(s) \phi_{2}(s) d s & =\left(\frac{\beta}{\beta-1}\right)^{p}\left[t^{\frac{p}{\beta}} \int_{0}^{t} \phi_{2}(s) s^{-\frac{p}{\beta}} d s+t^{p} \int_{t}^{b} \phi_{2}(s) s^{-p} d s\right] \\
& =\frac{p}{\beta^{\prime}}\left(\frac{\beta}{\beta-1}\right)^{p} t^{\frac{p}{\beta}} \int_{0}^{t} s^{\frac{p}{\beta^{\prime}}-1} \int_{s}^{b} \phi_{2}(y) y^{-p} d y d s .
\end{aligned}
$$

Thus, (5.3) implies (5.4).

Conversely, assume (5.4) is satisfied. By [12, Theorem 3.8], we have

$$
\left(T_{\beta} f\right)^{* *}(t) \leq 2\left(T_{\beta} f^{* *}\right)(t), \quad t \in I_{b}
$$

Therefore, in order to obtain (5.3), we need only show

$$
\int_{I_{b}}\left(T_{\beta} f^{* *}\right)(t)^{p} \phi_{2}(t) d t \leq C \int_{I_{b}} f^{* *}(s)^{p} \phi_{1}(s) d s, \quad f \in \mathfrak{M}_{+}\left(I_{b}\right) .
$$

In the remainder of the proof we suppose $b<\infty$; the argument in case $b=\infty$ is even simpler.

Now, an elementary calculation yields

$$
\left(T_{\beta} f^{* *}\right)(t)^{p} \leq \begin{cases}2^{\frac{p}{\beta}} t^{-\frac{p}{\beta}} \sup _{t \leq s<\frac{b}{2}} s^{\frac{p}{\beta}} f^{* *}(s)^{p}, & 0<t<\frac{b}{2}, \\ 2^{\frac{p}{\beta}} f^{* *}\left(\frac{b}{2}\right)^{p}, & \frac{b}{2} \leq t<b .\end{cases}
$$

Further, when $0<t<\frac{b}{2}$, one has

$$
\sup _{t \leq y<\frac{b}{2}} y^{\frac{p}{\beta}} f^{* *}(y)^{p} \leq 2^{p+1} \int_{t}^{b} f^{* *}(s)^{p} s^{\frac{p}{\beta}-1} d s, \quad t \in I_{\frac{b}{2}},
$$

since, given $t<y<\frac{b}{2}$,

$$
\begin{aligned}
\int_{t}^{b} f^{* *}(s)^{p} s^{\frac{p}{\beta}-1} d s & \geq \int_{y}^{2 y} f^{* *}(s)^{p} s^{\frac{p}{\beta}-1} d s \\
& \geq f^{* *}(2 y)^{p} y^{\frac{p}{\beta}} \log 2 \geq\left(2^{-p} \log 2\right) y^{\frac{p}{\beta}} f^{* *}(y) .
\end{aligned}
$$


Thus,

$$
\begin{aligned}
\int_{0}^{\frac{b}{2}}\left(T_{\beta} f^{* *}\right)(t)^{p} \phi_{2}(t) d t & \leq 2^{p+1} \int_{0}^{b} t^{\frac{p}{\beta}} \int_{t}^{b} f^{* *}(s)^{p} s^{\frac{p}{\beta}-1} d s \phi_{2}(t) d t \\
& =2^{p+1} \int_{I_{b}} f^{* *}(s)^{p} s^{\frac{p}{\beta}-1} \int_{0}^{s} \phi_{2}(t) t^{\frac{p}{\beta}} d t d s .
\end{aligned}
$$

We conclude

$$
\int_{0}^{\frac{b}{2}}\left(T_{\beta} f^{* *}\right)(t)^{p} \phi_{2}(t) d t \leq C \int_{I_{b}} f^{* *}(s)^{p} \phi_{1}(s) d s,
$$

provided

$$
\int_{I_{b}} f^{* *}(s)^{p} s^{\frac{p}{\beta}-1} \int_{0}^{s} \phi_{2}(y) y^{\frac{p}{\beta}} d y d s \leq 2^{-p-1} C \int_{I_{b}} f^{* *}(s)^{p} \phi_{1}(s) d s,
$$

which according to $[\mathbf{1 9}$, Theorem 3.3] is equivalent to (5.4).

Again, taking $t=\frac{b}{2}$ in (5.4), there follows

$$
\begin{aligned}
2^{-p} \frac{\beta^{\prime}}{p} & \leq\left(\frac{b}{2}\right)^{\frac{p}{\beta}} \int_{0}^{\frac{b}{2}} s^{\frac{p}{\beta^{\prime}}-1} d s \int_{\frac{b}{2}}^{b} \phi_{2}(y) y^{-p} d y \\
& \leq\left(\frac{b}{2}\right)^{\frac{p}{\beta}} \int_{0}^{\frac{b}{2}} s^{\frac{p}{\beta^{\prime}}-1} \int_{s}^{b} \phi_{2}(y) y^{-p} d y d s \\
& \leq K \int_{0}^{\frac{b}{2}} s^{p-1} \int_{s}^{b} \phi_{1}(y) y^{-p} d y d s \\
& \leq K\left[\int_{0}^{\frac{b}{2}} \phi_{1}(s) d s+\int_{\frac{b}{2}}^{b} \phi_{1}(s) d s\right] .
\end{aligned}
$$

Altogether, then, with $C=\frac{p}{\beta^{\prime}} 2^{p\left[1+\frac{1}{\beta}\right]} K$,

$$
\begin{aligned}
\int_{\frac{b}{2}}^{b}\left(T_{\beta} f^{* *}\right)(s)^{p} \phi_{2}(s) d s & \leq 2^{\frac{p}{\beta}} \int_{\frac{b}{2}}^{b} f^{* *}\left(\frac{b}{2}\right)^{p} \phi_{2}(s) d s \\
& \leq C\left[\int_{0}^{\frac{b}{2}} f^{* *}\left(\frac{b}{2}\right)^{p} \phi_{1}(s) d s+\int_{\frac{b}{2}}^{b} f^{* *}\left(\frac{b}{2}\right)^{p} \phi_{1}(s) d s\right] \\
& \leq 2^{p} C \int_{I_{b}} f^{* *}(s) \phi_{1}(s) d s .
\end{aligned}
$$

This completes the proof. 


\section{Applications and Examples}

The Marcinkiewicz space, $M(\alpha, X)$, is the Köthe dual of the original Lorentz space, $\Lambda\left(\alpha^{\prime}, X\right), \alpha^{\prime}=\frac{\alpha}{\alpha-1}$. Accordingly, one obtains

Theorem 6.1. Let $\left(X_{1}, \mu_{1}\right)$ and $\left(X_{2}, \mu_{2}\right)$ be $\sigma$-finite measure spaces for which $\mu_{1}\left(X_{1}\right)=\mu_{2}\left(X_{2}\right)=b$. Suppose the linear operator $T$ satisfies

$$
T: \Lambda\left(\alpha, X_{1}\right) \rightarrow \Lambda\left(\alpha, X_{2}\right) \text { and } T: \Lambda\left(\beta, X_{1}\right) \rightarrow \Lambda\left(\beta, X_{2}\right),
$$

for indices $\alpha$ and $\beta$, with $1<\alpha<\beta<\infty$. Define the r.i. norms, $\varrho_{i}$, on $\mathfrak{M}\left(X_{i}\right)$ in terms of the r.i. norms $\bar{\varrho}_{i}$ on $\mathfrak{M}\left(I_{b}\right)$ by

$$
\varrho_{i}(f):=\bar{\varrho}_{i}\left(f^{*}\right)
$$

and suppose

$$
\Lambda\left(\alpha, X_{i}\right) \cap \Lambda\left(\beta, X_{i}\right) \subset L_{\varrho_{i}}\left(X_{i}\right) \subset \Lambda\left(\alpha, X_{i}\right)+\Lambda\left(\beta, X_{i}\right), \quad i=1,2 .
$$

Then,

$$
T: L_{\varrho_{1}}\left(X_{1}\right) \rightarrow L_{\varrho_{2}}\left(X_{2}\right),
$$

whenever

$$
S_{\alpha^{\prime}}: L_{\bar{\varrho}_{2}^{\prime}}\left(I_{b}\right) \rightarrow L_{\bar{\varrho}_{1}^{\prime}}\left(I_{b}\right) \text { and } T_{\beta^{\prime}}: L_{\bar{\varrho}_{2}^{\prime}}\left(I_{b}\right) \rightarrow L_{\bar{\varrho}_{1}^{\prime}}\left(I_{b}\right),
$$

where $\alpha^{\prime}=\frac{\alpha}{\alpha-1}, \beta^{\prime}=\frac{\beta}{\beta-1}$.

Theorem A'. Let $\left(X_{1}, \mu_{1}\right)$ and $\left(X_{2}, \mu_{2}\right)$ be $\sigma$-finite measure spaces with $\mu_{1}\left(X_{1}\right)=\mu_{2}\left(X_{2}\right)=b$. Fix the indices $\alpha$ and $\beta, 1<\alpha<\beta<\infty$. Suppose $A_{1}$ and $A_{2}$ are Young functions satisfying

$$
\Lambda\left(\alpha, X_{i}\right) \cap \Lambda\left(\beta, X_{i}\right) \subset L_{A_{i}}\left(X_{i}\right) \subset \Lambda\left(\alpha, X_{i}\right)+\Lambda\left(\beta, X_{i}\right), \quad i=1,2 .
$$

Then, given any linear operator $T$ such that

$$
T: \Lambda\left(\alpha, X_{1}\right) \rightarrow \Lambda\left(\alpha, X_{2}\right) \text { and } T: \Lambda\left(\beta, X_{1}\right) \rightarrow \Lambda\left(\beta, X_{2}\right),
$$

one has

$$
T: L_{A_{1}}\left(X_{1}\right) \rightarrow L_{A_{2}}\left(X_{2}\right),
$$

whenever

$$
\begin{aligned}
& \int_{b^{-1}}^{t} \frac{\tilde{A}_{1}(s)}{s^{\alpha^{\prime}+1}} d s \leq \frac{\tilde{A}_{2}(K t)}{t^{\alpha^{\prime}}}, \\
& \int_{t}^{\infty} \frac{\tilde{A}_{1}(s)}{s^{\beta^{\prime}+1}} d s \leq \frac{\tilde{A}_{2}(K t)}{t^{\beta^{\prime}}},
\end{aligned}
$$

in which $\tilde{A}_{i}$ is the Young function complementary to $A_{i}, i=1,2, \alpha^{\prime}=$ $\frac{\alpha}{\alpha-1}, \beta^{\prime}=\frac{\beta}{\beta-1}$ and the constant $K>0$ is independent of $t>b^{-1}$. 
In particular, the first condition in (6.2) is necessary and sufficient in order that

$$
S_{\alpha^{\prime}}: L_{\tilde{A}_{2}}\left(I_{b}\right) \rightarrow L_{\tilde{A}_{1}}\left(I_{b}\right),
$$

while the second condition is necessary and sufficient for

$$
T_{\beta^{\prime}}: L_{\tilde{A}_{2}}\left(I_{b}\right) \rightarrow L_{\tilde{A}_{1}}\left(I_{b}\right) \text {. }
$$

Theorem B'. Let $\left(X_{1}, \mu_{1}\right)$ and $\left(X_{2}, \mu_{2}\right)$ be $\sigma$-finite measure spaces, with $\mu_{1}\left(X_{1}\right)=\mu_{2}\left(X_{2}\right)=b$. Fix the indices $\alpha$ and $\beta$ satisfying $1<\alpha<\beta<$ $\infty$. Suppose the index $p, 1<p<\infty$, and the nontrivial weight functions, $\phi_{1}$ and $\phi_{2}$, are such that

$$
\Lambda\left(\alpha, X_{i}\right) \cap \Lambda\left(\beta, X_{i}\right) \subset \Gamma_{p, \phi_{i}}\left(X_{i}\right) \subset \Lambda\left(\alpha, X_{i}\right)+\Lambda\left(\beta, X_{i}\right), \quad i=1,2 .
$$

Assume, in addition,

$$
\int_{0}^{1} \phi_{i}(t) t^{-p} d t=\int_{1}^{\infty} \phi_{i}(t) d t=\infty, \quad i=1,2
$$

if $b=\infty$. Then, given any linear operator $T$ for which

$$
T: \Lambda\left(\alpha, X_{1}\right) \rightarrow \Lambda\left(\alpha, X_{2}\right) \text { and } T: \Lambda\left(\beta, X_{1}\right) \rightarrow \Lambda\left(\beta, X_{2}\right),
$$

one has

$$
T: \Gamma_{p, \phi_{1}}\left(X_{1}\right) \rightarrow \Gamma_{p, \phi_{2}}\left(X_{2}\right)
$$

whenever

$$
\int_{0}^{t} \psi_{1}(s) d s+t^{\frac{p^{\prime}}{\alpha^{\prime}}} \int_{t}^{b} \psi_{1}(s) s^{-\frac{p^{\prime}}{\alpha^{\prime}}} d s \leq K \int_{0}^{t} s^{p^{\prime}-1} \int_{s}^{b} \psi_{2}(y) y^{-p^{\prime}} d y d s,
$$

$$
t^{\frac{p^{\prime}}{\beta^{\prime}}} \int_{0}^{t} \psi_{1}(s) s^{-\frac{p^{\prime}}{\beta^{\prime}}} d s+t^{p^{\prime}} \int_{t}^{b} \psi_{1}(y) y^{-p^{\prime}} d y \leq K \int_{0}^{t} s^{p^{\prime}-1} \int_{s}^{b} \psi_{2}(y) y^{-p^{\prime}} d y d s
$$

in which

$$
\psi_{i}(t):=\frac{t^{p^{\prime}+p-1} \int_{0}^{t} \phi_{i}(s) d s+\int_{t}^{b} \phi_{i}(s) s^{-p} d s}{\left(\int_{0}^{t} \phi_{i}(s) d s+t^{p} \int_{t}^{b} \phi_{i}(s) s^{-p} d s\right)^{p^{\prime}+1}}, \quad i=1,2,
$$

$p^{\prime}=\frac{p}{p-1}, \alpha^{\prime}=\frac{\alpha}{\alpha-1}, \beta^{\prime}=\frac{\beta}{\beta-1}$ and the constant $K>0$ is independent of $t \in I_{b}$.

In particular, the first condition in (6.3) is necessary and sufficient in order that

$$
S_{\alpha}: \Gamma_{p^{\prime}, \psi_{2}}\left(I_{b}\right) \rightarrow \Gamma_{p^{\prime}, \psi_{1}}\left(I_{b}\right),
$$

while the second one is necessary and sufficient for

$$
T_{\beta}: \Gamma_{p^{\prime}, \psi_{2}}\left(I_{b}\right) \rightarrow \Gamma_{p^{\prime}, \psi_{1}}\left(I_{b}\right) .
$$

We next consider what happens when $X_{1}=X_{2}=X, \mu_{1}=\mu_{2}=\mu$ and $\varrho_{1}=\varrho_{2}=\varrho$ in our theorems. 
Theorem 6.2. Let $(X, \mu)$ be a $\sigma$-finite measure space, with $\mu(X)=b$. Define the r.i. norm $\varrho$ on $\mathfrak{M}(X)$ in terms of the r.i. norm $\bar{\varrho}$ on $\mathfrak{M}\left(I_{b}\right)$ by

$$
\varrho(f):=\bar{\varrho}\left(f^{*}\right) .
$$

Fix indices $\alpha$ and $\beta$ satisfying $1<\alpha<\beta<\infty$. Then, the conditions

$$
S_{\alpha}: L_{\bar{\varrho}}\left(I_{b}\right) \rightarrow L_{\bar{\varrho}}\left(I_{b}\right) \text { and } T_{\beta}: L_{\bar{\varrho}}\left(I_{b}\right) \rightarrow L_{\bar{\varrho}}\left(I_{b}\right)
$$

are equivalent to $L_{\varrho}(X)$ being an interpolation space between $M(\alpha, X)$ and $M(\beta, X)$. Again, the conditions

$$
S_{\alpha^{\prime}}: L_{\bar{\varrho}^{\prime}}\left(I_{b}\right) \rightarrow L_{\bar{\varrho}^{\prime}}\left(I_{b}\right) \text { and } T_{\beta^{\prime}}: L_{\bar{\varrho}^{\prime}}\left(I_{b}\right) \rightarrow L_{\bar{\varrho}^{\prime}}\left(I_{b}\right)
$$

are equivalent to $L_{\varrho}(X)$ being an interpolation space between $\Lambda(\alpha, X)$ and $\Lambda(\beta, X)$; as usual, $\alpha^{\prime}=\frac{\alpha}{\alpha-1}$ and $\beta^{\prime}=\frac{\beta}{\beta-1}$.

Proof: Taking $X_{1}=X_{2}=X, \mu_{1}=\mu_{2}=\mu$ and $\varrho_{1}=\varrho_{2}=\varrho$ in the Dmitriev-Kreln-Peetre Theorem, it is seen that (6.4) implies $L_{\varrho}(X)$ is an interpolation space between $M(\alpha, X)$ and $M(\beta, X)$. As for the converse, we observe that, according to [13, Theorem 2.3], the conditions (6.4) are equivalent to $L_{\varrho^{\prime}}(X)$ being an interpolation space for both $\left(L_{1}(X), \Lambda\left(\alpha^{\prime}, X\right)\right)$ and $\left(\Lambda\left(\beta^{\prime}, X\right), L_{\infty}(X)\right)$ which, in turn, amounts to $L_{\varrho}(X)$ being an interpolation space for $\left(L_{1}(X), M(\beta, X)\right)$ and $\left(M(\alpha, X), L_{\infty}(X)\right)$. But, $M(\alpha, X)$ and $M(\beta, X)$ are interpolation spaces for the couples $\left(L_{1}(X), M(\beta, X)\right)$ and $\left(M(\alpha, X), L_{\infty}(X)\right)$, respectively. This means that whenever $L_{\varrho}(X)$ is an interpolation space for $(M(\alpha, X)$, $M(\beta, X))$ it is also an interpolation space for $\left(L_{1}(X), M(\beta, X)\right)$ and $\left(M(\alpha, X), L_{\infty}(X)\right)$ and hence the conditions (6.4) hold.

The second assertion follows by an argument similar to the one above.

Theorem 6.3. Fix indices $\alpha$ and $\beta$ satisfying $1<\alpha<\beta<\infty$. Let $\varrho$ be an Orlicz norm or a Lorentz gamma norm on $\mathfrak{M}\left(I_{b}\right)$ having Boyd indices $i_{\bar{\varrho}}$ and $I_{\bar{\varrho}}$. Then, the following are equivalent:

(i) $S_{\alpha}: L_{\bar{\varrho}}\left(I_{b}\right) \rightarrow L_{\bar{\varrho}}\left(I_{b}\right)$;

(ii) $t^{\frac{1}{\alpha}} \bar{\varrho}\left(s^{-\frac{1}{\alpha}} \chi_{(t, b)}(s)\right) \leq C \varrho\left(\chi_{(0, t)}\right)$, with $C>0$ independent of $t \in I_{b}$;

(iii) $\alpha<i_{\bar{\varrho}}$.

Again, the following are equivalent:

(iv) $T_{\beta}: L_{\bar{\varrho}}\left(I_{b}\right) \rightarrow L_{\bar{\varrho}}\left(I_{b}\right)$;

(v) $t^{\frac{1}{\beta}} \bar{\varrho}\left(s^{-\frac{1}{\beta}} \chi_{(0, t)}(s)\right) \leq C \varrho\left(\chi_{(0, t)}\right)$, with $C>0$ independent of $t \in I_{b}$;

(vi) $I_{\bar{\varrho}}<\beta$. 
Proof: The equivalence of (i) and (ii) and of (iv) and (v) has been established in Theorems A and B. Again, the estimate

$$
\begin{aligned}
\left(S_{\alpha} f\right)(t) & =t^{-\frac{1}{\alpha}} \sup _{0<s \leq t} s^{\frac{1}{\alpha}} f^{*}(s) \\
& \leq t^{-\frac{1}{\alpha}} \sup _{0<s \leq t} s^{\frac{1}{\alpha}-1} \int_{0}^{s} f^{*}(y) d y \\
& \leq t^{-\frac{1}{\alpha}} \sup _{0<s \leq t} \int_{0}^{s} f^{*}(y) y^{\frac{1}{\alpha}-1} d y \\
& =\left(P_{\alpha} f^{*}\right)(t),
\end{aligned}
$$

together with Theorem 2.2, give (i) from (iii). A similar argument shows (vi) entails (iv).

Now, in [16, Theorem 11.8, pp. 90-91], the assertions (i) implies (iii) and (iv) implies (vi) are proved in the case $\varrho$ is an Orlicz norm. The argument used is quite general and, indeed, works for Lorentz gamma norms as well, in view of Theorem 2.2.

Remark 6.4. When the norm $\varrho$ in Theorem 6.3 is an Orlicz norm, $\varrho_{A}$, (ii) and (v), together, are equivalent to the conditions (1.1) from the interpolation theorem of Zygmund. In view of (iii) and (vi), and [3, Theorem 1], the weak-type assumptions of that theorem can be replaced by the less demanding restricted weak-type requirements

$$
T: \Lambda(\alpha, X) \rightarrow M(\alpha, X) \text { and } T: \Lambda(\beta, X) \rightarrow M(\beta, X) .
$$

The next example shows the conditions (1.2) and (6.1) in Theorems 1.1 and 6.1, respectively, are not necessary to guarantee the conclusions of those theorems.

Example 6.5. Fix $\beta, 1<\beta<\infty$. One readily verifies that

$$
T_{\beta}: \Lambda(\alpha, q, I) \rightarrow \Lambda(\alpha, q, I), \quad I=(0,1),
$$

if and only if $1<\alpha<\beta$ and $1 \leq q \leq \infty$ or $\alpha=\beta$ and $q=\infty$, in which case $\Lambda(\beta, q, I)=M(\beta, I)$. Again,

$$
S_{\beta^{\prime}}: \Lambda\left(\alpha^{\prime}, q, I\right) \rightarrow \Lambda\left(\alpha^{\prime}, q, I\right)
$$

if and only if $1<\alpha<\beta$ and $1 \leq q \leq \infty$ or $\alpha=\beta$ and $q=\infty$, when $\Lambda\left(\beta^{\prime}, q, I\right)=M\left(\beta^{\prime}, I\right)$. 
However, for the linear operator $T$ given by

$$
f \rightarrow t^{-\frac{1}{\beta}}\left(\log \frac{1}{t}\right)^{-\frac{1}{\gamma}} \int_{0}^{1} f(s) d s, \quad 1<\gamma<\infty,
$$

with associate operator $T^{\prime}$ sending

$$
g \rightarrow \int_{0}^{1} g(t) t^{-\frac{1}{\beta}}\left(\log \frac{1}{t}\right)^{-\frac{1}{\gamma}} d t
$$

one has

$$
T: \Lambda(\beta, q, I) \rightarrow \Lambda(\beta, q, I)
$$

if and only if $\gamma<q \leq \infty$, and

$$
T^{\prime}: \Lambda\left(\beta^{\prime}, q, I\right) \rightarrow \Lambda\left(\beta^{\prime}, q, I\right)
$$

if and only if $1 \leq q<\gamma^{\prime}$.

Our goal now is to use results already obtained to study operators, $T$, satisfying more general conditions than those considered so far, namely,

$$
T: M\left(\alpha_{1}, X_{1}\right) \rightarrow M\left(\alpha_{2}, X_{2}\right) \text { and } T: M\left(\beta_{1}, X_{1}\right) \rightarrow M\left(\beta_{2}, X_{2}\right)
$$

where $1<\alpha_{i}<\beta_{i}<\infty, i=1,2$. In particular, we seek an explicit connection between norms $\varrho_{1}$ and $\varrho_{2}$ in an inequality of the form

$$
\varrho_{2}(T f) \leq C \varrho_{1}(f) .
$$

This connection is supplied by Theorem 2.4 .

Theorem 6.6. Let $\left(X_{1}, \mu_{1}\right)$ and $\left(X_{2}, \mu_{2}\right)$ be $\sigma$-finite measure spaces with $\mu_{1}(X)=\mu_{2}(X)=b$. For $i=1,2$, fix indices $\alpha_{i}$ and $\beta_{i}$ satisfying $1<\alpha_{i}<\beta_{i}<\infty$. Given an r.i. norm, $\bar{\varrho}$, on $\mathfrak{M}\left(I_{b}\right)$ define the r.i. functionals

$$
\begin{aligned}
\varrho_{1}(g):=\bar{\varrho}\left(t^{-1} \sup _{0<s \leq t^{\gamma_{1}}} s^{\frac{1}{\alpha_{1}}} g^{*}(s)+\sup _{t^{\gamma_{1}}<s<b} s^{\frac{1}{\beta_{1}}} g^{*}(s)\right), \\
\\
\quad g \in \mathfrak{M}\left(X_{1}\right), \quad \frac{1}{\gamma_{1}}=\frac{1}{\alpha_{1}}-\frac{1}{\beta_{1}},
\end{aligned}
$$

and

$$
\begin{aligned}
\varrho_{2}(h):=\bar{\varrho}\left(t^{-1} \sup _{0<s \leq t^{\gamma_{2}}} s^{\frac{1}{\alpha_{2}}} h^{*}(s)+\sup _{t^{\gamma_{2}<s<b}} s^{\frac{1}{\beta_{2}}} h^{*}(s)\right), \\
\\
\quad h \in \mathfrak{M}\left(X_{2}\right), \quad \frac{1}{\gamma_{2}}=\frac{1}{\alpha_{2}}-\frac{1}{\beta_{2}} .
\end{aligned}
$$


Then, $\varrho_{1}$ and $\varrho_{2}$ are equivalent to r.i. norms on $\mathfrak{M}\left(X_{1}\right)$ and $\mathfrak{M}\left(X_{2}\right)$, respectively. Moreover, if the linear operator $T$ satisfies

$$
T: M\left(\alpha_{i}, X_{i}\right) \rightarrow M\left(\beta_{i}, X_{i}\right), \quad i=1,2,
$$

and if $i_{\bar{\varrho}}>1$, one has

$$
\varrho_{2}(T f) \leq C \varrho_{1}(f),
$$

where $C>0$ is independent of $f \in \mathfrak{M}\left(X_{1}\right), \varrho_{1}(f)<\infty$.

Proof: Theorem 2.4 ensures the inequality

$$
\begin{aligned}
\bar{\varrho}\left(k \left(t,(T f)^{*} ; M\left(\alpha_{2}, X_{2}\right)\right.\right. & \left.\left., M\left(\beta_{2}, X_{2}\right)\right)\right) \\
& \leq C \bar{\varrho}\left(k\left(t, f^{*} ; M\left(\alpha_{1}, X_{1}\right), M\left(\beta_{1}, X_{1}\right)\right)\right)
\end{aligned}
$$

in which $C>0$ is independent of $f \in \mathfrak{M}\left(X_{1}\right)$. Again, $i_{\bar{\varrho}}>1$ means

$$
\bar{\varrho}\left(P g^{*}\right) \approx \bar{\varrho}\left(g^{*}\right), \quad g \in \mathfrak{M}(I),
$$

so, (6.6) implies (6.5).

Acknowledgement. We would like to thank the referee for her/his careful and critical reading of the paper and in particular for pointing out an error in our original proof of Theorem B.

\section{References}

[1] C. Bennett and R. Sharpley, "Interpolation of operators", Pure and Applied Mathematics 129, Academic Press, Inc., Boston, MA, 1988.

[2] D. W. BoyD, The Hilbert transform on rearrangement invariant spaces, PhD. Thesis, University of Toronto (1966).

[3] D. W. Boyd, Indices of function spaces and their relationship to interpolation, Canad. J. Math. 21 (1969), 1245-1254. DOI: 10.4153/CJM-1969-137-x.

[4] M. Carro, L. Pick, J. Soria, and V. D. Stepanov, On embeddings between classical Lorentz spaces, Math. Inequal. Appl. 4(3) (2001), 397-428. DOI: $10.7153 / \mathrm{mia}-04-37$.

[5] A. Cianchi, A sharp embedding theorem for Orlicz-Sobolev spaces, Indiana Univ. Math. J. 45(1) (1996), 39-65. DOI: 10.1512/iumj. 1996.45.1958.

[6] A. Cianchi, R. Kerman, B. Opic, and L. Pick, A sharp rearrangement inequality for the fractional maximal operator, Studia Math. 138(3) (2000), 277-284. 
[7] V. I. Dmitriev And S. G. KreǏn, Interpolation of operators of weak type, Anal. Math. 4(2) (1978), 83-99. DOI: 10.1007/ BF02116975.

[8] A. Gogatishvili and R. Kerman, The rearrangement-invariant space $\Gamma_{p, \phi}$, Positivity, to appear.

[9] A. Gogatishvili, B. Opic, And L. Pick, Weighted inequalities for Hardy-type operators involving suprema, Collect. Math. 57(3) (2006), 227-255.

[10] A. Gogatishvili And L. Pick, Discretization and anti-discretization of rearrangement-invariant norms, Publ. Mat. 47(2) (2003), 311-358. DOI : 10.5565/PUBLMAT_47203_02.

[11] R. A. Hunt, On $L(p, q)$ spaces, Enseignement Math. (2) 12 (1966), 249-276.

[12] R. Kerman and L. Pick, Optimal Sobolev imbeddings, Forum Math. 18(4) (2006), 535-570. DOI: 10.1515/FORUM.2006.028.

[13] R. Kerman and L. Pick, Optimal Sobolev imbedding spaces, Studia Math. 192(3) (2009), 195-217. DOI: 10.4064/sm192-3-1.

[14] J. Lindenstrauss And L. Tzafriri, "Classical Banach spaces. I. Sequence spaces", Ergebnisse der Mathematik und ihrer Grenzgebiete 92, Springer-Verlag, Berlin-New York, 1977; "Classical Banach spaces. II. Function spaces", Ergebnisse der Mathematik und ihrer Grenzgebiete 97, Springer-Verlag, Berlin-New York, 1979.

[15] G. G. Lorentz, Some new functional spaces, Ann. of Math. (2) 51 (1950), 37-55.

[16] L Maligranda, "Orlicz spaces and interpolation", Seminários de Matemática [Seminars in Mathematics] 5, Universidade Estadual de Campinas, Departamento de Matemática, Campinas, 1989.

[17] C. J. Neugebauer, Weighted norm inequalities for averaging operators of monotone functions, Publ. Mat. 35(2) (1991), 429-447. DOI : 10.5565 /PUBLMAT_35291_07.

[18] E. SAWYer, Boundedness of classical operators on classical Lorentz spaces, Studia Math. 96(2) (1990), 145-158.

[19] V. D. Stepanov, Integral operators on the cone of monotone functions, J. London Math. Soc. (2) 48(3) (1993), 465-487. DOI: $10.1112 / \mathrm{j} 1 \mathrm{~ms} / \mathrm{s} 2-48.3 .465$.

[20] A. Zygmund, "Trigonometric series", 2nd ed., Vols. I, II, Cambridge University Press, New York, 1959. 
Ron Kerman:

Department of Mathematics

Brock University

500 Glenridge Ave.

St. Catharines, Ontario

Canada L2S 3A1

E-mail address: rkerman@brocku.ca

Colin Phipps:

Department of Applied Mathematics

University of Waterloo

200 University Ave. W.

Waterloo, Ontario

Canada N2L 3G1

E-mail address: cphipps@uwaterloo.ca

Luboš Pick:

Department of Mathematical Analysis

Faculty of Mathematics and Physics

Charles University

Sokolovská 83

18675 Praha 8

Czech Republic

E-mail address: pick@karlin.mff.cuni.cz

Primera versió rebuda el 15 de novembre de 2011, darrera versió rebuda el 14 de març de 2013. 